\title{
Toward accurate antenna measurements using multi-probe systems
}

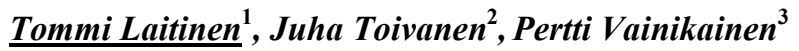 \\ ${ }^{1,3}$ Aalto University School of Electrical Engineering, Department of Radio Science and Engineering, P.O. Box 13000, \\ 00076 AALTO, Finland, ${ }^{1}$ Tommi.Laitinen@aalto.fi, ${ }^{3}$ Pertti.Vainikainen@aalto.fi \\ ${ }^{2}$ Saïd Business School, University of Oxford, The Queen's College, Oxford, OX1 4AW, United Kingdom, \\ Juha.Toivanen@queens.ox.ac.uk
}

\begin{abstract}
In this paper a few important aspects related to spherical multi-probe antenna measurements and their future research and development needs are discussed. In particular, the significance of the test zone field compensation technique as an enabler for accurate antenna measurements with multi-probe systems is addressed.
\end{abstract}

\section{Introduction}

Antenna measurements have been a subject of extensive research efforts over several decades. Various techniques have been developed for different, specific antenna measurement needs. Typically, the antenna far-field radiation properties have been of interest, and a far-field measurement has been the natural choice for obtaining this information. Due to the requirements for a large measurement distance for large antennas at high frequencies, several alternative measurement techniques have been developed. Some of the most well known alternative techniques here are near-field antenna measurement and compact range techniques.

A compact range can be considered to be a large probe that directly measures the relative far-field pattern in the near field of the antenna under test (AUT). Hence, ideally speaking, there is no need for compensating any near-field effects that may be present, because the reflector converts the near field of the AUT at the position of the reflector to a relative far-field value in the direction where the reflector locates.

On the contrary, with the near-field techniques one does not directly measure a relative far field of the AUT in the direction of the probe but rather a weighted sum of the field over the volume of the probe. With the near-field techniques, it is generally necessary to first gather sufficient information over the complete measurement surface, and then to perform a mathematical transformation for obtaining the far-field radiation pattern. It is sometimes assumed that the probe directly measures a field component of the near field of the AUT, and in this case the mathematical transformation to obtain the far field is simply a near-field to far-field transformation. However, in many cases [1] it cannot be assumed that the probe would be measuring a field component of the near field, and it then becomes necessary to perform probe correction in order to compensate for the effect of the probe pattern. The transformation to be performed in this case can be referred to, for example, either as a near-field to far-field transformation including probe correction or, more directly, a probe signal to far-field transformation. Through particularly the use of the probe correction, the near-field techniques have the reputation of being an accurate technique for antenna pattern characterization.

One of the most recent big trends in the antenna measurement field has been the development of spherical multiprobe antenna measurement systems. The growth of the mobile telecommunications industry during the last 20 years created a need particularly for rapid antenna testing, and this paved the way for these multi-probe systems [2]-[4]. Although a significant concern with the multi-probe systems among the industry has been the scattering from the neighboring probes in such systems and the influence of this on the estimate of the far field pattern, the requirement for the antenna pattern accuracy in the mobile terminal antenna testing was not so severe that this would have prevented the multi-probe system becoming common. However, it is quite obvious that this specific concern has slowed down the pace by which the multi-probe systems have become common among many other antenna measurement applications where the accuracy requirements are higher.

The purpose of this paper is to discuss a few important aspects related to spherical multi-probe antenna measurements and their future research and development needs. In particular, the significance of the test zone field compensation technique as an enabler for accurate antenna measurements with multi-probe systems is addressed. 


\section{Multi-probe systems}

\subsection{Probe compensation in single-probe systems vs. test zone field compensation at multi-probe systems}

In [5] a technique known as test zone field (TZF) compensation technique is presented for performing compensation of scattered fields from surrounding structures in spherical near-field antenna measurements. Although the investigations of that paper are performed on single-probe systems, the results are fundamental and significant for the development of particularly the multi-probe antenna measurement technology. In view of the concern on the influence of the scattered field from the neighboring probes in a multi-probe system on the errors in the transformed farfield pattern, the TZF compensation technique presented in [5] provides a crucial theoretical basis for fully compensating these errors. The procedure of the antenna measurements (by either a single or multi-probe system) based on the TZF compensation is, in a sense, analogous to that of the well-known, traditional spherical near-field measurements with probe correction, and this issue deserves to be discussed now. A brief comparison of these two measurement methods is presented in the following.

In the traditional near-field antenna measurements with probe correction [6], the free-space assumption allows the use of the translation theorems for spherical wave functions, and this provides the possibility for determining the probe response constants analytically from the known probe pattern and the measurement distance in wavelengths. These probe response constants represent the responses of the probe toward the different outward-propagating spherical mode fields in the test zone of the AUT. Additional application of the spherical wave rotation functions allows establishing the free-space transmission formula for the probe received signal as a function of the spherical vector wave coefficients of the radiated field of the AUT. The aim is then to solve this transmission formula for those spherical vector wave coefficients computationally from the measured probe signals around the AUT and the known probe pattern, and AUT far-field pattern is ultimately obtained with the found coefficients. These traditional spherical nearfield antenna measurements with probe correction hence require the probe pattern measurement and subsequently the AUT pattern measurement with the known probe.

In the TZF compensation, free-space assumption is made only for the test zone of the AUT. Due to this, there is not any practical possibility for determining the probe response constants or establishing a transmission formula analytically. In the case of multi-probe systems, the constants analogous to the "probe response constants" in singleprobe systems have been referred to as "multi-probe system response constants" in [3], and these constants are determined by a TZF measurement with a known probe. Hence, calculating them through the translation theorems for spherical vector wave functions is not required or it cannot be done. After determining the multi-probe system response constants in the first step by the TZF measurement with a known probe, the second step involves the AUT pattern measurement.

\subsection{Experiments}

Recently, after having first introduced in [5], the TZF compensation technique has been theoretically further examined in [7], and also experimentally validated in [8], [9] and [10]. Papers [7] and [9] do not directly concern multiprobe systems but they provide additional proof on the validity of the TZF compensation technique as a method for compensating the effect of the scattered fields. Papers [8] and [10] in turn are directly related to the application of the probe array compensation on multi-probe range.

The specific system where the tests were made in [10] is shown in Fig. 1. The system consists of 64 probes arranged on a spherical surface at the distance of approximately $1 \mathrm{~m}$ from the center of the sphere. The TZF compensation was demonstrated by performing in the first step the over-the-air probe array calibration measurement with the TZF probe to determine the multi-probe system response constants for each 64 channels of the systems. In the second step the actual AUT measurement was performed, and with the known multi-probe system response constants the AUT pattern with full TZF compensation could be obtained. 


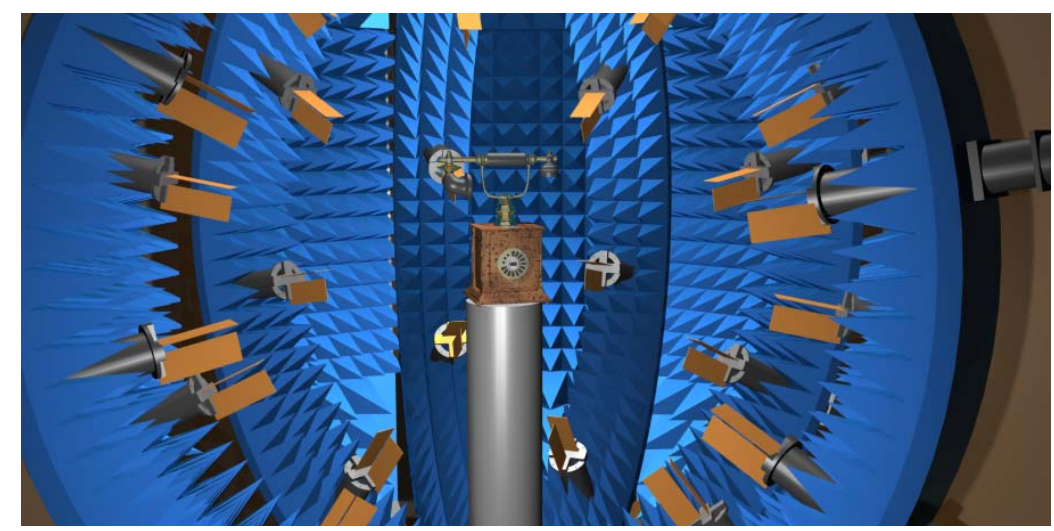

Fig. 1. Illustration of the 64-probe measurement system.

The results presented in [10] provide a proof that the test zone field compensation principle works. The obtained results are in a very good agreement with reference measurements performed in the DTU-ESA Spherical Near-field Antenna Test Facility [11], and hence they show that accurate antenna pattern measurements are possible on multiprobe systems.

\subsection{Research and development needs}

Some practical and also more theoretical challenges and research needs for multi-probe systems, however, do exist. Some of these are discussed now.

In the first step of the application of the test zone field compensation, a TZF probe measurement in the AUT test zone is performed. Related to this measurement an issue to be made clear is the condition(s) under which this measurement does not provide sufficient information for the determining the AUT pattern in the second step. One must make sure that the multi-probe system response constants are obtained for all spherical modes. Obviously this issue becomes particularly important for electrically large test zone dimensions and for ultra-wideband measurement applications. One additional issue in the TZF measurement is the TZF probe back-lobe pattern uncertainty. The TZF probe pattern information on the complete $4 \pi$ solid angle is exploited in the determination of the multi-probe system response constants from the TZF field measurement, and hence any uncertainty in the TZF probe pattern, that is particularly imminent in the back-lobe direction, will directly influence on the accuracy of the multi-probe system response constants. This is clearly an issue that requires further investigations.

The computational algorithms required in the second step of the test zone field compensation to determine the spherical vector wave coefficients of the AUT comprise another issue that requires further investigations. By the choice of a proper probe, for example, so-called first-order probe and/or a proper scanning technique, the computational requirements do not need to become a problem in the first step of the application of the test zone field compensation, but the situation is not so straight-forward in the second step. Iterative algorithms are likely to be required for reaching the sufficiently low computational complexity for electrically very large AUTs in the second step.

One practical concern is the validity time period of the multi-probe system response constants. In order to avoid frequent TZF probe measurement, it would be beneficial to have a mechanically stable chamber that is insensitive to any moisture and temperature variations. It is noted that as the absorbers are not generally required to be used, it becomes fair to ask what would be the optimum material to be used in the multi-probe ranges based on the test zone field compensation. Clearly, however, some absorbing properties are desirable as the multiple reflections between the AUT and the probes inside the chamber cannot be compensated by the test zone field compensation technique.

\section{Conclusions}

Multi-probe antenna measurement systems have a well-established position in mobile telecommunications industry, and particularly in the testing of mobile terminals. In this paper, a few important issues related to the development of spherical multi-probe antenna measurement systems have been discussed and some challenges and research needs addressed. The conclusion of this paper is that multi-probe systems with test zone field compensation capability have a great potential to become to the main stream antenna test technology in also many such applications where the utmost far-field pattern determination accuracy is required. This will require further research and development efforts on several topic areas. 


\section{Acknowledgments}

T. Laitinen would like to thank Academy of Finland (decision notification no. 129055) for the financial support of this work.

\section{References}

1. A. Yaghjian, "An overview of near-field antenna measurements," IEEE Transactions on Antennas and Propagation, Vol. 34, No. 1, Jan. 1986, pp. 30-45.

2. http://www.satimo.com/

3. T. Laitinen, "Advanced Spherical Antenna Measurements," Doctoral thesis, Helsinki University of Technology, Finland, Dec. 2005, 63 p. (+ attached papers).

4. J. Toivanen, "Measurement methods for mobile terminal antenna performance," Doctoral thesis, Aalto University, Finland, Sept. 2010, 76 p. (+ attached papers).

5. D. N. Black and E. B. Joy, "Test zone field compensation," IEEE Transactions on Antennas and Propagation, vol. 43, pp. 362-368, Apr. 1995.

6. J. E. Hansen (ed.), Spherical Near-Field Antenna Measurements. Peter Peregrinus, Ltd., London, 1988.

7. R. J. Pogorzelski, "Extended probe instrument calibration (EPIC) for accurate spherical near-field antenna measurements," IEEE Transactions on Antennas Propagation, vol. 57, pp. 3366-3371, Oct. 2009.

8. J. Toivanen, T. Laitinen, S. Pivnenko, and L. Nyberg, "Calibration of multi-probe antenna measurement system using test zone field compensation," in The $3^{\text {rd }}$ European Conference on Antennas and Propagation (EuCAP'09), Berlin, Germany, Mar. 23-27, 2009, pp. 2916-2920.

9. R. J. Pogorzelski, "Experimental demonstration of the extended probe instrument calibration (EPIC) technique," IEEE Transactions on Antennas Propagation, vol. 58, No. 6, pp. 2093-2097, June 2010.

10. J. Toivanen, T. Laitinen, and P. Vainikainen, "Modified test zone field compensation for small-antenna measurements," IEEE Transactions on Antennas Propagation, vol. 58, No. 11, pp. 3471-3479, Nov. 2010.

11. http://www.dtu.dk/centre/ems/English/research/facilities.aspx 